\title{
Status of branched endovascular aortic arch repair
}

\author{
Theodorus M. van Bakel ${ }^{1,2,3 \#}$, Hector W. de Beaufort ${ }^{1,2 \#}$, Santi Trimarchi ${ }^{2,4}$, Massimiliano M. Marrocco- \\ Trischitta $^{2}$, Jean Bismuth ${ }^{5}$, Frans L. Moll ${ }^{1}$, Himanshu J. Patel ${ }^{6}$, Joost A. van Herwaarden ${ }^{1}$ \\ ${ }^{1}$ Department of Vascular Surgery, University Medical Center Utrecht, Utrecht, The Netherlands; ${ }^{2}$ Thoracic Aortic Research Center, Policlinico San \\ Donato IRCCS, University of Milan, San Donato Milanese, Italy; ${ }^{3}$ Department of Surgery, Frankel Cardiovascular Center, University of Michigan, \\ Ann Arbor, MI, USA; ${ }^{4}$ Department of Biomedical Sciences for Health, University of Milan, Milan, Italy; ${ }^{5}$ Houston Methodist DeBakey Heart \& \\ Vascular Center, Houston, TX, USA; ${ }^{6}$ Department of Cardiac Surgery, Frankel Cardiovascular Center, University of Michigan, Ann Arbor, MI, USA \\ \#These authors contributed equally to this work. \\ Correspondence to: Theodorus M. van Bakel. 2800 Plymouth Road Building 20-211W, Ann Arbor, MI, 48105, USA. Email: vanbakel@med.umich.edu.
}

\begin{abstract}
Multiple medical device manufacturers are developing branched endografts for thoracic endovascular aortic repair (TEVAR), to provide a minimally invasive alternative for the treatment of aortic arch pathologies in patients who are deemed unfit for open or hybrid arch repair. Different branched endografts have been introduced, with varying number, size and orientation of the branches that redirect flow to the supra-aortic arteries. We present an overview of the currently investigated devices and review their outcomes. The results of branched TEVAR are promising, yet stroke remains the predominant periprocedural concern. For now, these procedures should be limited to select expert centers where the design and deployment procedure of branched endografts can be further developed to reduce the risk of stroke.
\end{abstract}

Keywords: Thoracic endovascular aortic repair (TEVAR); branched TEVAR; aortic arch repair; stent graft design

Submitted Feb 13, 2018. Accepted for publication Mar 13, 2018.

doi: 10.21037/acs.2018.03.13

View this article at: http://dx.doi.org/10.21037/acs.2018.03.13

\section{Introduction}

Open surgical repair is the gold standard for the treatment of aortic arch pathologies (1). Due to the invasive nature of the procedure, which involves sternotomy or thoracotomy, hypothermic circulatory arrest and cardiopulmonary bypass, many patients are deemed unfit for surgery (2). Hybrid aortic arch repairs, such as the "frozen elephant trunk" technique, reduce cardiac ischemia times, but are still invasive procedures associated with postoperative stroke rates up to $13 \%$ and 30 -day mortality rates up to $12 \%$ (3). Thoracic endovascular aortic repair (TEVAR) could be a minimally invasive alternative for patients who are deemed unfit for open or hybrid surgical repair. Yet, the origin of the supra-aortic arteries and aortic arch angulation pose anatomical challenges for the deployment of endografts $(4,5)$. As a result, the outcomes of TEVAR in combination with extensive supra-aortic interventions or chimney techniques show substantial rates of postoperative stroke, type I endoleak and retrograde type A dissection (6).
Multiple medical device manufacturers are now developing branched endografts for aortic arch deployment, extending the application of TEVAR to the proximal aorta. In the following, we review the literature on the technical characteristics and outcomes of branched TEVAR in the aortic arch and discuss the challenges and future perspectives of endovascular arch repair.

\section{Current techniques and outcomes of branched TEVAR}

The PubMed database was searched for studies on branched endovascular repair of aortic arch pathologies (zone 0-2). Original reports were included if the operative techniques and postoperative outcomes were reported. If multiple reports described (partly) the same patient cohort, the most recent report was selected. A total of 14 studies were included, describing endovascular treatment of 302 patients with branched endografts (7-20). Overall proportions were calculated with a meta-analysis of proportions in $\mathrm{R}$ version 
3.4.3, using the R package "meta" (21).

\section{Branched endograft designs}

Technical characteristics of the endografts that were described in the included studies are reported in Table 1. Figure 1 presents the single-branched endografts, Figure 2 presents the multi-branched endografts. W.L. Gore (Flagstaff, AZ, USA) and Medtronic Vascular (Santa Rosa, CA, USA) have developed single-branched endografts that are connected to the target vessel using a bridging stent $(7,16)$. The main difference between the designs of these devices is that the W.L. Gore device has an internal branch with a retrograde orientation and the Medtronic device has volcano-shaped opening without an internal branch. Endospan (Herzlia, Israel) and MicroPort Medical Co., Ltd. (Shanghai, China) developed single-branched endografts with a unibody design that can be combined with one or more fenestrations to accommodate perfusion of additional supra-aortic arteries $(9,18)$. Bolton Medical, (Sunrise, FL, USA) and Cook Medical (Bloomington, IN, USA) developed double-branched endografts for zone 0 deployment that feature anterior facing internal branches that are connected to the brachiocephalic trunk (BCT) and left common carotid artery (LCCA) with bridging stents $(8,14,20)$. Both endograft systems are individually made to fit the patient's anatomy, the main differences between the endograft designs are the position and size of the two internal branches. In the Bolton design, the internal branches have the same size and are positioned side-byside, while in the Cook design, the internal branch to the LCCA is often smaller and positioned diagonally behind the internal branch to the BCT. Another important difference is that the Bolton system includes the bridging stents, while the Cook system is not delivered with bridging stents. This means that with the Cook system, additional bridging stents need to be used outside their instructions for use. The Inoue Stent Graft has a unibody design without internal branches. This endograft has been developed at the PTMC institute (Kyoto, Japan) and is individually constructed from a woven Dacron polyester fabric. The branches are sewn upon the main body at the location of the target vessels (12).

\section{Deployment procedures}

The deployment procedure for branched endografts is similar to the procedure for regular TEVAR, yet additional guidewires are inserted for deployment of the branch components. Through-and-through access is optional, depending on the patient's anatomy and physician's preference (16). Inoue et al. reported the first total endovascular aortic arch repair in 1999 (17). The triplebranched Inoue Stent Graft was inserted transfemorally with one large caliber sheath (24 F). The branch sections of the endograft were pulled into the target vessels by traction wires that were inserted via the bilateral brachial arteries and LCCA using small caliber sheaths (5-7 F). The deployment procedure of modular branched endografts is similar; however, additional components need to be introduced via the supra-aortic arteries to connect the branches in the main body to the target vessels. The brachial, axillary and carotid arteries can be used as access vessels to advance guidewires and bridging components into the aorta, depending on local vascular anatomy and preference of the physician.

\section{Outcomes of branched TEVAR}

Table 2 presents the outcomes of the included studies. The overall technical success rate was high and the endografts provided good patency during follow-up (94\% and $96 \%$, respectively). The overall rate of retrograde type A dissection was $3.3 \%$ and overall early mortality was $6.1 \%$, which is low considering the fact that the vast majority of patients had multiple comorbidities and were deemed unfit for open surgical repair. The results from Haulon et al. (8) highlighted a steep learning curve, even for the highly skilled endovascular specialist, with an early mortality rate of $30 \%$ in the first ten cases. An improvement followed in the next 28 cases, with a mortality rate of $7.1 \%$. Postoperative stroke is the Achilles' heel of endovascular arch repair, with an overall combined incidence of $14 \%$. Multi-branched endograft deployments were associated with high stroke rates. Tazaki et al. reported stroke rates of $33 \%$ with the double-branched and $40 \%$ for the triple-branched Inoue Stent Graft (12). Spear et al. reported a $14.8 \%$ stroke rate with the double-branched endograft from Cook (14) and Czerny et al. reported a combined stroke rate of $20 \%$ with the Bolton double-branched endograft (20). Guidewire manipulation in the arch and supra-aortic arteries, which are frequently burdened by atherosclerosis $(7,8)$, is most likely the cause of embolic stroke in these cases $(14,20)$.

\section{Challenges of branched TEVAR}

A recent CT-based anatomical feasibility study estimated that about $70 \%$ of patients with arch aneurysms after 


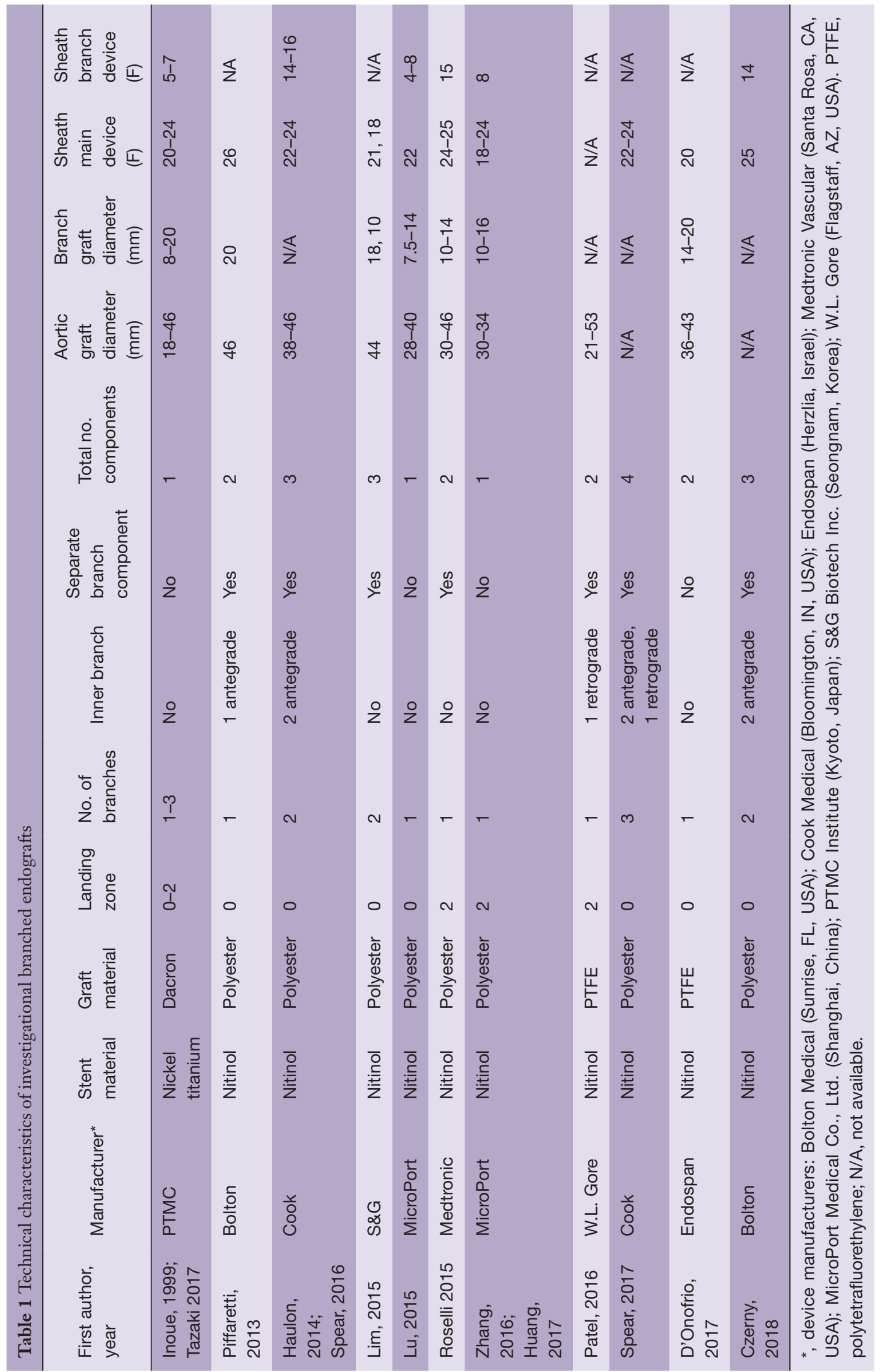


A

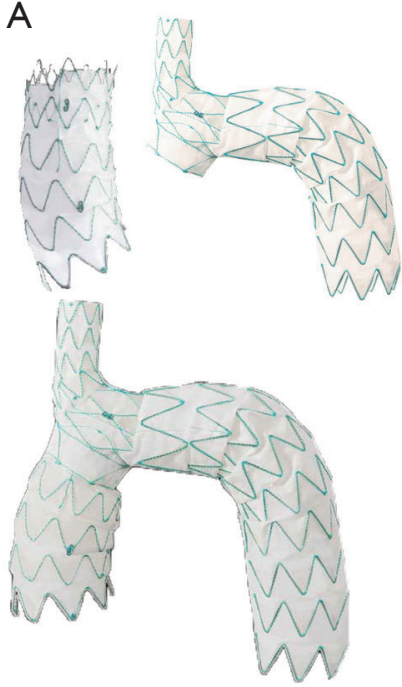

B

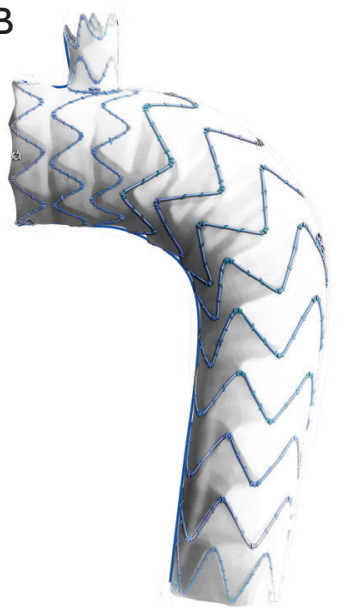

C

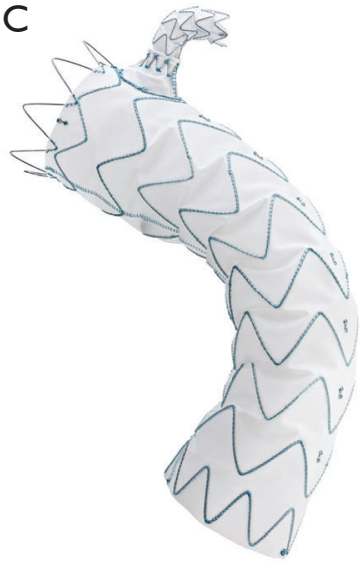

Thoracic Branch Stent graft is an investigational device, limited by law to investigational use only. Not approved for sale in the US or OUS: exclusively for clinical investigation. (92018 Medtronic. All Rights Reserved.
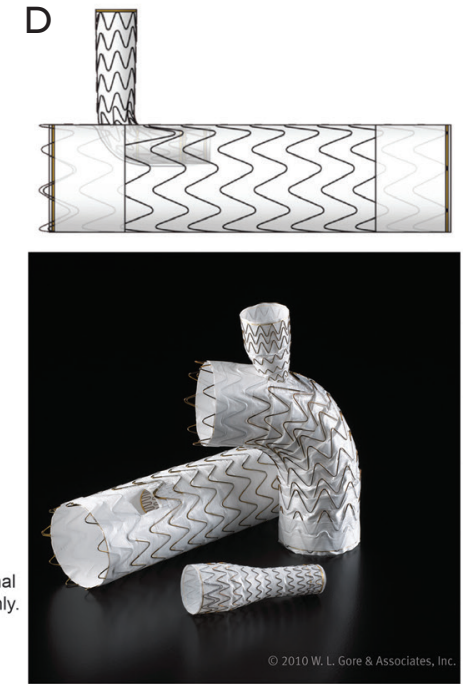

Figure 1 Single-branched endografts. (A) Nexus ${ }^{\mathrm{TM}}$ Stent Graft System for zone 0 from Endospan (Herzlia, Israel), this endograft is customizable with an additional fenestration for the left common carotid artery. Image provided courtesy of Endospan; (B) Castor ${ }^{\mathrm{TM}}$ branched endograft from MicroPort Medical Co., Ltd. (Shanghai, China), customizable with two additional fenestrations. Image provided courtesy of MicroPort Medical; (C) thoracic branch stent graft from Medtronic Vascular (Santa Rosa, CA, USA). Image provided courtesy of Medtronic Vascular; (D) GORE ${ }^{\circledR} \mathrm{TAG}^{\circledast}$ thoracic endoprosthesis with retrograde internal branch from W.L. Gore (Flagstaff, AZ, USA). Image provided courtesy of W.L. Gore \& Associates, Inc.
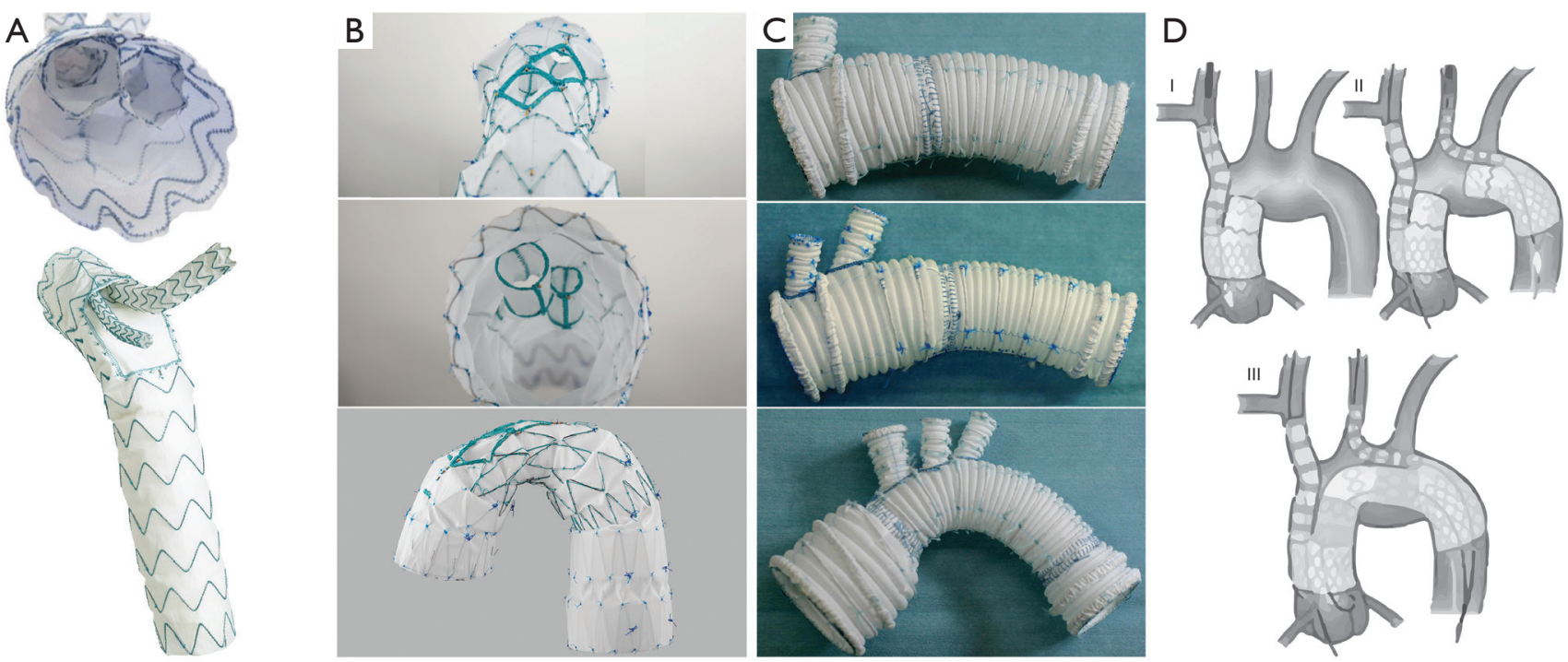

Figure 2 Multi-branched endografts. (A) Zone 0 endograft from Bolton Medical, permission for use was granted by Bolton Medical, Sunrise, Florida; (B) zone 0 endograft from Cook Medical, permission for use granted by Cook Medical, Bloomington, Indiana; (C) three versions of the Inoue Stent Graft from the PTMC institute (Kyoto, Japan), image from Tazaki et al. (12); (D) three component solution for zone 0 deployment from S\&G Biotech, Inc. (Seongnam, Korea), image from Lim et al. (19). 


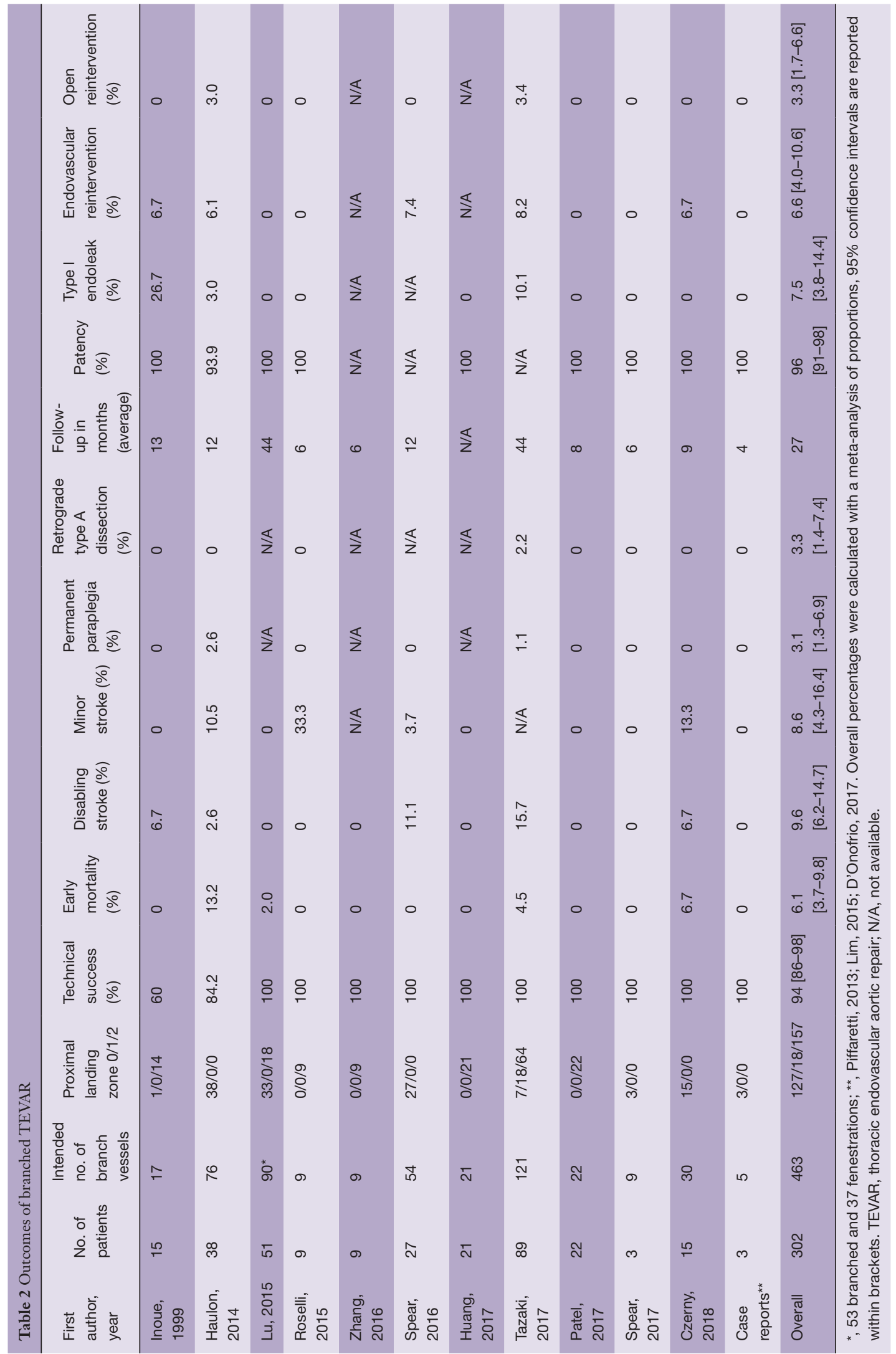


surgical replacement of the ascending aorta are feasible for endovascular treatment with one of the currently investigated double-branched devices (22). This is particularly interesting, as redo sternotomies add significant risks for postoperative complications (23). Still, the majority of multi-branched endografts for aortic arch deployment are individually made, which takes several weeks and limits the application of total endovascular arch repair to elective cases. The case report from D'Onofrio et al. presented a successful off-the-shelf solution for total endovascular arch repair, using the single-branched modular Nexus system from Endospan (Herzlia, Israel) (9). Before deployment of this single-branched endograft, the patient underwent an extra-anatomic reconstruction of the supra-aortic arteries with a right common carotid to LSA bypass and reimplantation of the LCCA on the graft. A recent computational study from our group analyzed the postoperative hemodynamic outcomes of different endograft designs for zone 0 repair (24). We found that a single-branched design resulted in reduced perfusion of the supra-aortic arteries with elevated blood shear rates, compared to double-branched alternatives. The case report from Lim et al. presented a double-branched off-theshelf solution for zone 0 endovascular arch repair, using bifurcated endografts in the BCT and LCCA that were connected with a bridging component in the aortic arch (19). An important limitation of this technique is the need to introduce large caliber devices via the carotid arteries. These devices may damage the arterial wall of the carotid arteries, increasing postoperative stroke risks. Additionally, the hemodynamic displacement forces in the arch need to be taken into account (5), as they pose a challenge for proximal sealing and integrity of modular endografts during follow-up (25).

\section{Our perspective}

Branched TEVAR is a promising extension of the armamentarium of endovascular specialists. However, this technically challenging procedure requires optimal equipment and endovascular experience and is associated with a steep learning curve. Preoperative planning is vital for a successful branched TEVAR procedure and should include imaging with echocardiographic gated computed tomography angiography (CTA) to reduce artefacts generated by prior surgery material and cardiac motion (26). The images are then processed using software-assisted centerline reconstructions to acquire accurate measurements of the luminal diameter, arch angulation and proximal and distal landing zones lengths. These measurements should be discussed within the endovascular team, including the endograft manufacturer, to determine the feasibility of branched TEVAR and to select the optimal endograft. Complex endovascular procedures, such as branched TEVAR, should be performed in hybrid operating rooms that combine an optimal open surgical environment with advanced imaging capabilities, in an effort to facilitate endovascular navigation and increase accuracy of endograft deployment. Moreover, the use of CTA image fusion with live fluoroscopy has been shown to reduce radiation exposure and contrast injection during complex repairs (27). Further development of endograft design and deployment procedure is needed to reduce stroke rates following branched TEVAR. Routine use of transcranial Doppler ultrasonography can help to identify the procedural aspects most associated with cerebral embolization (28). Perera et al. recently reported that robotic catheter placement resulted in significantly less cerebral embolization during TEVAR compared with manual techniques (29). Additional carbon dioxide flushing of the endograft prior to insertion could reduce the risk of air embolism during deployment (30). The use of cerebral protection devices, which have proven to be effective at reducing embolization in transcatheter aortic valve replacement (31), could also be effective for branched arch TEVAR. Additionally, patient-specific computational fluid dynamics analysis can help to predict the hemodynamic outcomes of different treatment scenarios and aid endograft selection $(24,32)$.

\section{Conclusions}

Multiple medical device manufacturers are developing branched endografts for aortic arch deployment, providing a minimally invasive solution for patients who are deemed unfit for open or hybrid arch repair. The results of branched TEVAR are promising, yet stroke remains the predominant periprocedural concern of total endovascular arch repair. For now, these procedures should be limited to select expert centers where the design and deployment procedure of branched endografts can be further developed to reduce stroke risks.

\section{Acknowledgements}

This work was generously supported by the Bob and Ann Aikens Aortic Grants Program, the David Hamilton Fund 
and the Phil Jenkins Breakthrough Fund in Cardiac Surgery at the University of Michigan Frankel Cardiovascular Center.

\section{Footnote}

Conflicts of Interest: Prof. HJ Patel serves as consultant and co-patent holder with W.L. Gore and consultant for Medtronic Inc. and Terumo Inc.; Prof. S Trimarchi serves as consultant and speaker for W.L. Gore and Medtronic Inc.; Dr. JA van Herwaarden serves as consultant and speaker for Bolton Medical, Cook Medical and Philips Health Care; Dr. J Bismuth serves as consultant and speaker for W.L. Gore. All authors declare no conflicts of interest related to the contents of the manuscript.

\section{References}

1. Hiratzka LF, Bakris GL, Beckman JA, et al. 2010 ACCF/AHA/AATS/ACR/ASA/SCA/SCAI/SIR/STS/ SVM guidelines for the diagnosis and management of patients with Thoracic Aortic Disease: a report of the American College of Cardiology Foundation/American Heart Association Task Force on Practice Guidelines, A. Circulation 2010;121:e266-369.

2. Lioupis C, Abraham CZ. Results and challenges for the endovascular repair of aortic arch aneurysms. Perspect Vasc Surg Endovasc Ther 2011;23:202-13.

3. Shrestha M, Beckmann E, Krueger H, et al. The elephant trunk is freezing: The Hannover experience. J Thorac Cardiovasc Surg 2015;149:1286-93.

4. Marrocco-Trischitta MM, de Beaufort HW, Secchi F, et al. A geometric reappraisal of proximal landing zones for thoracic endovascular aortic repair according to aortic arch types. J Vasc Surg 2017;65:1584-90.

5. Marrocco-Trischitta MM, van Bakel TM, Romarowski RM, et al. The Modified Arch Landing Areas Nomenclature (MALAN) Improves Prediction of Stent Graft Displacement Forces: Proof of Concept by Computational Fluid Dynamics Modelling. Eur J Vasc Endovasc Surg 2018;55:584-92.

6. Andrasi TB, Grossmann M, Zenker D, et al. Supra-aortic interventions for endovascular exclusion of the entire aortic arch. J Vasc Surg 2017;66:281-97.e2.

7. Roselli EE, Arko FR, Thompson MM. Results of the Valiant Mona LSA early feasibility study for descending thoracic aneurysms. J Vasc Surg 2015;62:1465-71.e3.

8. Haulon S, Greenberg RK, Spear R, et al. Global experience with an inner branched arch endograft. J Thorac Cardiovasc Surg 2014;148:1709-16.

9. D'Onofrio A, Antonello M, Lachat M, et al. Endovascular treatment of aortic arch aneurysm with a single-branched double-stage stent graft. J Thorac Cardiovasc Surg 2017;154:e75-7.

10. Piffaretti G, Rivolta N, Fontana F, et al. Aortic arch aneurysm repair with a new branched device. J Vasc Surg 2013;57:1664-7.

11. Zhang T, Jiang $\mathrm{W}$, Lu H, et al Thoracic Endovascular Aortic Repair Combined with Assistant Techniques and Devices for the Treatment of Acute Complicated Stanford Type B Aortic Dissections Involving Aortic Arch. Ann Vasc Surg 2016;32:88-97.

12. Tazaki J, Inoue K, Higami H, et al. Thoracic endovascular aortic repair with branched Inoue Stent Graft for arch aortic aneurysms. J Vasc Surg 2017;66:1340-8.e5.

13. Huang $\mathrm{H}$, Jiao $\mathrm{Y}$, Zhang $\mathrm{Y}$, et al. Implantation of Unibody Single-Branched Stent Graft for Patients with Type B Aortic Dissections Involving the Left Subclavian Artery: 1-Year Follow-Up Outcomes. Cardiovasc Intervent Radiol 2017;40:1678-86.

14. Spear R, Haulon S, Ohki T, et al. Editor's Choice Subsequent Results for Arch Aneurysm Repair with Inner Branched Endografts. Eur J Vasc Endovasc Surg 2016;51:380-5.

15. Spear R, Clough RE, Fabre D, et al. Total Endovascular Treatment of Aortic Arch Disease Using an Arch Endograft with 3 Inner Branches. J Endovasc Ther 2017;24:534-8.

16. Patel HJ, Dake MD, Bavaria JE, et al. Branched Endovascular Therapy of the Distal Aortic Arch: Preliminary Results of the Feasibility Multicenter Trial of the Gore Thoracic Branch Endoprosthesis. Ann Thorac Surg 2016;102:1190-8.

17. Inoue $\mathrm{K}$, Hosokawa $\mathrm{H}$, Iwase $\mathrm{T}$, et al. Aortic Arch Reconstruction by Transluminally Placed Endovascular Branched Stent Graft. Circulation 1999;100:II316-21.

18. Lu Q, Feng J, Zhou J, et al. Endovascular repair by customized branched stent-graft: A promising treatment for chronic aortic dissection involving the arch branches. J Thorac Cardiovasc Surg 2015;150:1631-8.e5.

19. Lim JW, Choi CW, Her K, et al. Totally endovascular aortic arch repair by branched stent graft placement. J Vasc Surg Cases 2015;1:279-82.

20. Czerny M, Rylski B, Morlock J, et al. Orthotopic branched endovascular aortic arch repair in patients who cannot undergo classical surgery. Eur J Cardiothorac Surg 
2018;53:1007-12.

21. Schwarzer G, Carpenter JR, Rücker G. Meta-Analysis with R. 1st edition. Heidelberg: Springer, 2015.

22. Milne CP, Amako M, Spear R, et al. Inner-Branched Endografts for the Treatment of Aortic Arch Aneurysms After Open Ascending Aortic Replacement for Type A Dissection. Ann Thorac Surg 2016;102:2028-35.

23. Rylski B, Desai ND, Bavaria JE, et al. Type A aortic dissection after previous cardiac surgery: Results of an integrated surgical approach. Ann Thorac Surg 2014;97:1582-8; discussion 1588-9.

24. van Bakel TM, Arthurs CJ, van Herwaarden JA, et al. A computational analysis of different endograft designs for Zone 0 aortic arch repair. Eur J Cardiothorac Surg 2018. [Epub ahead of print].

25. Prasad A, To LK, Gorrepati ML, et al. Computational analysis of stresses acting on intermodular junctions in thoracic aortic endografts. J Endovasc Ther 2011;18:559-68.

26. Valente T, Rossi G, Lassandro F, et al. MDCT evaluation of acute aortic syndrome (AAS). Br J Radiol 2016;89:20150825.

Cite this article as: van Bakel TM, de Beaufort HW, Trimarchi S, Marrocco-Trischitta MM, Bismuth J, Moll FL, Patel HJ, van Herwaarden JA. Status of branched endovascular aortic arch repair. Ann Cardiothorac Surg 2018;7(3):406-413. doi: 0.21037/ acs.2018.03.13
27. Hertault A, Maurel B, Sobocinski J, et al. Impact of hybrid rooms with image fusion on radiation exposure during endovascular aortic repair. Eur J Vasc Endovasc Surg 2014;48:382-90.

28. Bismuth J, Garami Z, Anaya-Ayala JE, et al. Transcranial Doppler findings during thoracic endovascular aortic repair. J Vasc Surg 2011;54:364-9.

29. Perera AH, Riga CV, Monzon L, et al. Robotic Arch Catheter Placement Reduces Cerebral Embolization During Thoracic Endovascular Aortic Repair (TEVAR). Eur J Vasc Endovasc Surg 2017;53:362-9.

30. Rohlffs F, Tsilimparis N, Saleptsis V, et al. Air Embolism During TEVAR: Carbon Dioxide Flushing Decreases the Amount of Gas Released from Thoracic Stent-Grafts During Deployment. J Endovasc Ther 2017;24:84-8.

31. Giustino G, Sorrentino S, Mehran R, et al. Cerebral Embolic Protection During TAVR: A Clinical Event Meta-Analysis. J Am Coll Cardiol 2017;69:465-6.

32. van Bakel TMJ, Lau KD, Hirsch-Romano J, et al. PatientSpecific Modeling of Hemodynamics: Supporting Surgical Planning in a Fontan Circulation Correction. J Cardiovasc Transl Res 2018;11:145-55. 